\title{
Differences in total and truncal body fat mass in children using DXA and BIA method - multifactorial regression model
}

\author{
Elżbieta Jakubowska-Pietkiewicz ${ }^{1}$, Paulina Adamiecka ${ }^{1}$, Wojciech Fendler ${ }^{2,3}$, \\ Agnieszka Szadkowska ${ }^{4}$ \\ ${ }^{1}$ Department of Paediatric Propedeutics and Bone Metabolism Diseases, Medical University of Lodz, Poland \\ 2Department of Biostatistics ad Translational Medicine, Medical University of Lodz, Poland \\ ${ }^{3}$ Department of Radiation Oncology, Dana-Farber Cancer Institute, Harvard Medical School, Boston, MA, USA \\ ${ }^{4}$ Department of Pediatrics, Oncology, Hematology and Diabetology, Medical University of Lodz, Poland
}

\begin{abstract}
Objective: The objective of the study was to establish a model that would reduce the bioimpedance measurement error as compared with the densitometric result, in terms of total and segmental fatty tissue content in children.

Material and methods: The study consisted of 56 children, aged 6-18. All subjects underwent a densitometry examination (DXA) to assess total fatty tissue mass $(\%, \mathrm{~g})$, and percentage of fatty tissue on limbs and trunk. The bioelectric impedance (BIA) analyses were performed using a Tanita scale, considering identical measurement parameters.
\end{abstract}

Results: The average percent deviation between values obtained with densitometry and bioimpedance was $14.23 \pm 33.44 \%$ (median, 5.30; IQR, 1.35-10.9). The median of the mean absolute percentage error (MAPE) was 18.55 (median, 28.13; IQR, 18.47-38.88\%). The coefficient of determination $\left(R^{2}\right)$ of the simple linear regression of bioimpedance, as compared with densitometry, was $0.83(p<0.0001)$. The multi-factorial model significantly improved the estimation accuracy $(p<0.0001)$ - the mean estimation error was $6.11 \pm 29.30$ (median, -0.04 ; IQR, -7.97 through 10.96), while the MAPE median was $18.54 \pm 23.38$ (median, 9.89; IQR, 4.21-23.37). The final prediction model was based on percentage of parameters of overall fatty tissue content, percentage of fatty tissue on the right and left lower limb, and on the left upper limb.

Conclusions: The use of a multi-factorial regression model reduces the differences between results obtained using densitometry and those achieved with bioelectric impedance, in terms of overall and segmental fatty tissue content evaluation in children, improving the measurement accuracy.

Key words: body fat, densitometry examination, bioelectric impedance, children.

\section{Introduction}

Excess weight and obesity become a growing problem amongst the developmental age population. $\mathrm{Ng}$ et al. reported that between the years 1980 and 2013, the worldwide prevalence of overweight and obesity in children and adolescents has increased by $47.1 \%$ in both developed and developing countries [1]. Approximately 43 million children and adolescents are classified as obese, and $21-24 \%$ individuals are overweight. This may mean that a large number of the adult population may be at risk of obesity complications, such as metabolic syndrome, diabetes mellitus, cardiovascular diseases, various types of cancer, osteoarthritis, and even premature death from heart disease [2]. The most exposed to these complication risks are those children with abdominal obesity. In order to optimize the assessment of incidence of the problem of obesity and abdominal obesity, and to further monitor therapeu- 
tic processes, it is advised to evaluate the amount of fatty tissue in the body, and the amount of abdominal fatty tissue in children and youths. In everyday clinical practice, the assessment of obesity incidence is based mainly on anthropometric measurements of body height and weight as well as BMI calculation, whereas the evaluation of abdominal obesity is based on the measurement of the waist circumference, waist-to-hip ratio, and waist-to-height ratio. However, these methods do not allow an accurate evaluation of the fatty tissue contents in the body $[3,4]$. Total body water (TBW), derived from deuterium oxide dilution space, is a method to accurate evaluate body fatty tissue contents. Imaging techniques, such as computed tomography (CT), magnetic resonance (MR) imaging methods, MR imaging (MRI) and MR spectroscopy (MRS), are also used for that purpose $[4,5]$. However, densitometry is considered in many studies as a reference method for determining the amount of fatty tissue in the body [6,7]. Dual energy X-ray absorptiometry measurements (DXA) are used mainly for evaluation of bone mineral density but also for detailed body composition analyses $[7,8]$. This method enables the fatty tissue contents to be determined in the whole body (in $\mathrm{g}$ and \%) and in chosen segments, including limbs and trunk.

For over ten years, due to the DXA costs and limited availability for evaluation of fatty tissue amount, body composition analysis has been increasingly performed by using the bioelectrical impedance method (BIA). BIA is based on the principle that the body's electrical impedance is a function of distribution of water and electrolytes among various compartments in the body, i.e. total body water (TBW), intra-cellular body water (ICW), extra-cellular body water (ECW), body cell mass (BCM) in this body fat mass (FM), and fat-free body mass (FFM) [9]. It is a safe, non-invasive, and easy to perform procedure $[7,9,10]$. Our own results and other authors' observations indicate that the BIA method underestimates the over- all contents of fatty tissue in the body. Body segmental fatty tissue contents may be attained using BIA based on an eight-electrode measurement system. Contrarily to the DXA method, bioimpedance does not determine the fatty tissue contents on the trunk but estimates it based on measurements performed on limbs and trunk. Until present, there have been no comparisons of these twotrunk fatty tissue evaluation methods in children. Therefore, the objective of the study was to compare trunk fatty tissue evaluation results obtained using the DXA and BIA methods, and to design a model that would reduce the differences between BIA and DXA measurement results of overall and trunk fatty tissue contents in the developmental age population.

\section{Material and methods}

The study was conducted on a group of 56 children aged 6 to 18, which consisted of 23 girls and 33 boys. While performing tests, no children were subjected to chronic disease treatments. The patients' characteristics are summarized in Table 1. All subjects underwent anthropometric measurements of body weight and height as well as waist circumference (WC) according to accepted methods. Body weight was measured with a $0.1 \mathrm{~kg}$ accuracy, using a medical scale (Seca), while body height was measured with a $0.1 \mathrm{~cm}$ accuracy using a Martin anthropometer. The body mass index has been calculated using the formula: body mass (in $\mathrm{kg}$ )/height $\left(\mathrm{m}^{2}\right)$. The results were normalized. Waist circumference was measured twice using a tape measure, with an accuracy of $0.5 \mathrm{~cm}$. All subjects underwent a densitometry examination using double energy X-ray absorption measurements (DXA), and total body program, using a GE Lunar Prodigy device (Madison, USA). The measurement time was 12 minutes, the radiation dose was $1 \mathrm{mrem}$, and the measurement repeatability was $1 \%$. The study was conducted

Table 1. Characteristic of studied group

\begin{tabular}{|c|c|c|c|c|}
\hline \multirow[t]{2}{*}{ Factor } & \multicolumn{2}{|c|}{ Girls } & \multicolumn{2}{|c|}{ Boys } \\
\hline & $X \pm \mathrm{SD}$ & Range & $X \pm \mathrm{SD}$ & Range \\
\hline Age (years) & $14.1 \pm 2.8$ & $7.0-18.2$ & $13.5 \pm 2.9$ & $6.0-17.8$ \\
\hline Weight (kg) & $47.0 \pm 16.8$ & $22.2-96.1$ & $47.7 \pm 2.9$ & $18.1-81.7$ \\
\hline Height (cm) & $155.2 \pm 13.7$ & $120-174$ & $158 \pm 18$ & $115-185$ \\
\hline Waist circumference (cm) & $66 \pm 9$ & $54.0-98.0$ & $70.1 \pm 10.0$ & $54.5-96.5$ \\
\hline BMI $\left(k g / m^{2}\right)$ & $18.9 \pm 4.1$ & $12.5-31.7$ & $18.7 \pm 3.7$ & $13.6-28.1$ \\
\hline Weight SDS & $-0.12 \pm 1.68$ & $-2.09-5.65$ & $-0.35 \pm 1.05$ & $-2.98-2.18$ \\
\hline BMI SDS & $-0.19 \pm 1.35$ & $-1.77-4.44$ & $-0.27 \pm 1.05$ & $-2.55-2.77$ \\
\hline Waist circumference SDS & $0.32 \pm 1.48$ & $-1.76-5.19$ & $0.25 \pm 1.40$ & $-2.66-3.89$ \\
\hline
\end{tabular}


at the Regional Centre for Menopause and Osteoporosis of the Clinical Hospital No 3 of the Medical University in Lodz. Overall and segmental (for each of the four limbs and trunk) fatty tissue contents were determined (in grams and percentage). The bioelectric impedance (BIA) analysis was performed using a Tanita Corporation (Body Composition Analyser BC-418MA) scale with a built-in eight-electrode, four-limb system (Tanita Corporation, Tokyo, Japan). The test was performed 3 hours after meal and its duration was 2 minutes. The frequency of the current used was $50 \mathrm{KHz}$, with a $0.8-1 \mathrm{~mA}$ amperage. The tests were approved by the Bioethics Committee at the Medical University of Lodz.

\section{Statistical analysis}

Student $t$-test with independent variance estimation and paired Student $t$-test were used for comparisons of sex-depended values. For variables with an abnormal distribution, non-parametric counterparts of the above-mentioned tests were used (Mann-Whitney $U$ or Wilcoxon tests). Correlations were evaluated using the Pearson test, while the determination coefficient was calculated using linear regression, based on the least square regression curve matching. Method and model estimation accuracy evaluation was performed using the mean absolute percentage error (MAPE). Distribution normality for variables was tested using the Shapiro-Wilk test. The difference trends for various methods were determined using the Bland-Altman plot analysis. Statistical analyses were performed using the STATISTICA 8.0 PL StatSoft, USA) and Medcalc 9.3 (Medcalc, Mariekerke, Belgium) software suites.

\section{Results}

Table 2 presents the mean percentage of total and segmental fatty tissue contents in children, as recorded using the DXA and BIA methods. The $\mathrm{p}$ analysis indicates that bioimpedance overestimates the percentage values of fatty tissue of the upper body and underestimates these values for whole body and lower limbs evaluations.

In terms of overall body fatty tissue contents, the average percent deviation between values obtained with densitometry and with bioimpedance was $4.03 \pm 5.93 \%$ (median, 5.30; IQR, 0.65-8.5). The mean absolute percentage error (MAPE), for unadjusted bioimpedance results was $26.56 \pm 10.07$ (median, 23.10; IQR, 18.03-32.31\%). The observed coefficient of determination for the simple linear regression of bioimpedance, as compared with densitometry, was $\left(R^{2}=0.84 ; p<0.0001\right)$.

The measurement difference between DXA and BIA showed a positive correlation with BMI SDS $(r=0.64$; $p<0.0001)$, and standardized waist circumference $(r=$ $0.56 ; p<0.0001)$. There was no correlation observed with patients' sex $(p=0.152)$ and age $(p=0.236)$. Following a multi-factorial regression analysis, the best result was achieved with a single factorial model (Table 3).

The evaluation error was statistically significantly inferior for the model, as compared with results obtained as BIA unadjusted values ( $p<0.0001$; Figure 1$)$. Figure 2 shows the correlation between unadjusted percentage results of total body fatty tissue contents, values obtained after modification based on the formula, and DXA results.

When comparing trunk fatty tissue contents, the average percent deviation between values obtained with densitometry and with bioimpedance was $14.23 \pm 33.44 \%$

Table 2. Mean percentage of fat mass, total, and segmental in children using DXA and BIA method

\begin{tabular}{|l|c|c|c|}
\hline & DXA & BIA & $p$ value \\
\hline Total & $25.16 \pm 11.75$ & $21.13 \pm 7.12$ & $<0.0001$ \\
\hline RL & $28.42 \pm 11.13$ & $25.62 \pm 7.90$ & 0.0001 \\
\hline LL & $28.41 \pm 11.13$ & $25.87 \pm 7.76$ & 0.01 \\
\hline RA & $22.67 \pm 12.89$ & $28.99 \pm 7.28$ & $<0.0001$ \\
\hline LA & $22.68 \pm 12.89$ & $29.67 \pm 8.10$ & $<0.0001$ \\
\hline Trunk & $22.55 \pm 12.05$ & $16.91 \pm 7.23$ & $<0.0001$ \\
\hline
\end{tabular}

$R L$ - right leg; $L L$ - left leg; $R A$ - right arm; $L A-$ left arm

Table 3. Univariate regression results for total fatty tissue content evaluation basing on bioimpedance result. The B parameter corresponds to the slope of regression curve, while Beta is the partial correlation coefficient

\begin{tabular}{|l|c|c|c|}
\hline & Beta & B & $p$ value \\
\hline Intercept & - & -8.11 & 0.03 \\
\hline BIA results & 0.79 & 1.57 & 0.00001 \\
\hline
\end{tabular}




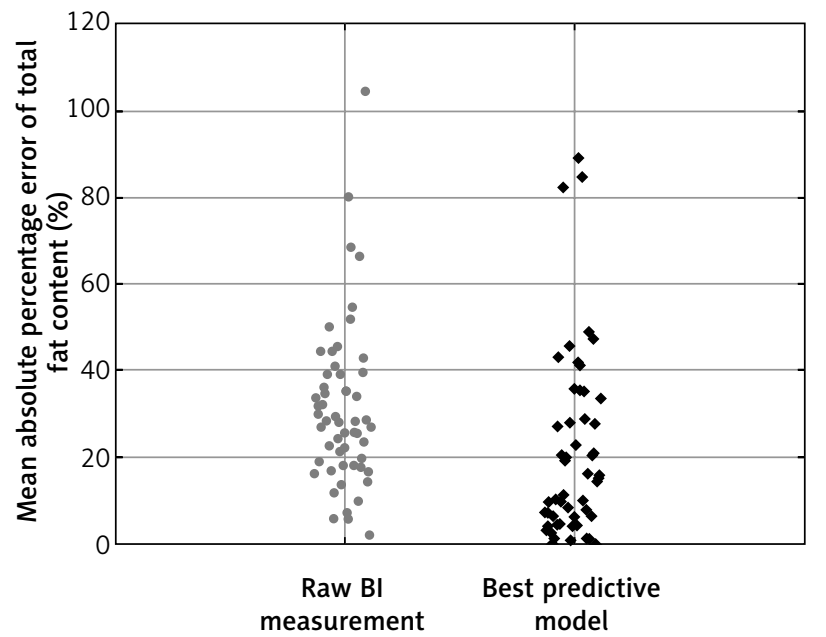

Figure 1. Values of mean absolute percentage error of raw bioimpedance and model-adjusted bioimpedance values for total fatty tissue content

(median 5.30\%; IQR, 1.35-10.9). The mean absolute percentage error (MAPE) for unadjusted bioimpedance results was $31.03 \% \pm 18.55$ (median, 28.13; IQR, 18.47$38.88 \%$ ). A high coefficient of determination was noted for the simple linear regression of bioimpedance, as compared with densitometry $\left(R^{2}=0.83 ; p<0.0001\right)$.

The difference between the densitometry and BIA results was insignificantly dependent upon sex $(M-7.46$ $\pm 5.89 ; \mathrm{M}-4.37 \pm 6.49 ; p=0.07)$ and was highly correlated with BMI SDS $(r=0.67 ; p<0.0001)$ and waist circumference SDS $(r=0.60 ; p<0.0001)$. There was no correlation observed with patient's age $(r=0.1 ; p=0.48)$. Table 3 presents parameters of the best multi-factorial regression model, as compared with the DXA method, for results obtained using the BIA method. When adjusting the best model created using a stepwise regression method, $R^{2}$ was found to be 0.89 , the mean estimation error was $6.11 \pm 29.30$ (median, -0.04; IQR, -7.97-10.96),

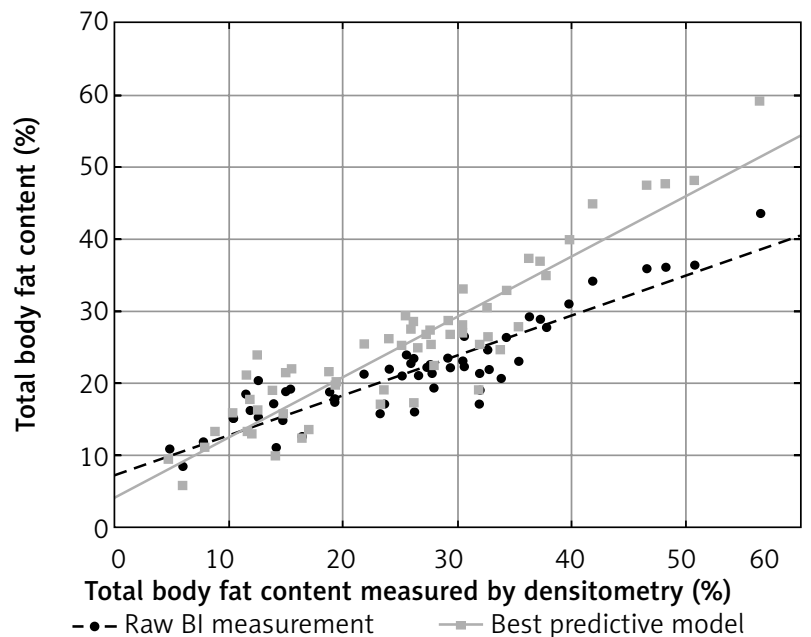

Figure 2. Correlation of raw and model-adjusted bioimpedance values with densitometry results for total fatty tissue content

while the MAPE was $18.54 \pm 23.38$ (median, 9.89; IQR, 4.21-23.37). The final prediction model was based on percentage of parameters of overall fatty tissue content, percentage of fatty tissue on the right lower limb, percentage of fatty tissue on the left lower limb, and percentage of fatty tissue on the left upper limb (Table 4). The evaluation error was statistically significantly inferior for the model, as compared with results obtained as BIA unadjusted values $(p<0.0001$, Figure 3$)$. Figure 4 shows the correlation between unadjusted percentage results of trunk fatty tissue contents, values obtained after modification based on the formula, and DXA results.

The correlation between values estimated using the multi-factorial regression model, unadjusted values, and effective values of trunk fatty tissue contents as determined upon densitometry, was statistically significant in both cases; however, the coefficient of correlation was superior for results obtained using the regression model

Table 4. Best multivariate regression model for trunk fatty tissue content calculation basing on bioimpedance results

\begin{tabular}{|l|c|c|c|}
\hline & Beta & B & $p$ value \\
\hline Intercept & & -6.37169 & 0.01 \\
\hline FAT\% - BI & 1.29743 & 2.19507 & 0.0001 \\
\hline RL_FAT\% BI & -1.15714 & -1.76439 & 0.01 \\
\hline LL_FAT\% BI & 1.16177 & 1.80351 & 0.01 \\
\hline LA_FAT\% & -0.42816 & -0.63720 & 0.0001 \\
\hline BMI SDS & 0.16461 & 1.49696 & 0.2 \\
\hline Waist circumference SDS & -0.10600 & -0.89656 & 0.3 \\
\hline RA\% FAT BI & 0.13349 & 0.22108 & 0.4 \\
\hline Male gender & 0.00942 & 0.22869 & 0.9 \\
\hline
\end{tabular}




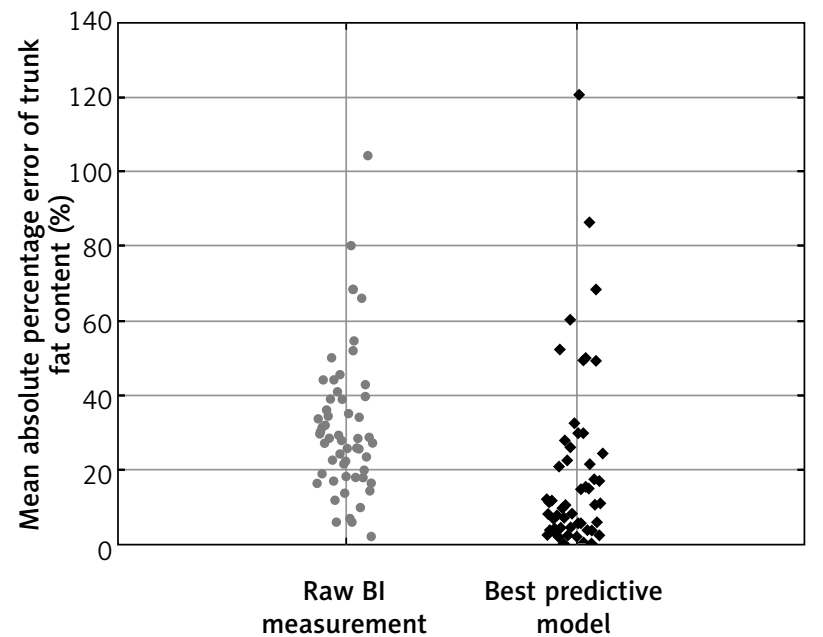

Figure 3. Values of mean absolute percentage error of raw bioimpedance and model-adjusted bioimpedance values for fatty tissue content within the trunk

$(r=0.95)$ than for unadjusted values $(r=0.90$; Figure 3$)$. Values of percentage difference between unadjusted bioimpedance results, model estimation, and densitometry results are presented in Figure 4. For unadjusted bioimpedance values, only $29 \%$ of the results were found to be within a $\pm 20 \%$ error, while for results obtained using the regression method, this rate was found to be $70 \%$.

\section{Discussion}

Due to the increasing problem of excess weight and obesity among the developmental age population, monitoring bodily fatty tissue contents has become a necessity. Anthropometric studies only allow an estimation of the fatty tissue content of the body. For a more accurate monitoring of therapeutic processes aimed at reducing the body fatty tissue contents, it is advisable to use more accurate diagnostic tools. Densitometry is considered to be one of the best methods for determining the amount of fatty tissue in the body [6,7]. In terms of determining the body composition, densitometry scans consider the total and segmental fatty tissue contents (including trunk fatty tissue), differentiate fatty tissue between android and gynoid type; therefore, densitometry is considered to be an optimal method [11-14]. However, its widespread use is not feasible.

BIA is more and more widely used due to the low cost of the test, its accessibility, high accuracy, and the lack of exposition to radiation [15-17]. The above features allow the method to be used for patient monitoring. However, one must note that results of studies comparing the BIA method with DXA indicate that BIA in slim subjects will tend to overestimate body fatty tissue, while in overweight subject, the fatty tissue contents will be underestimated as

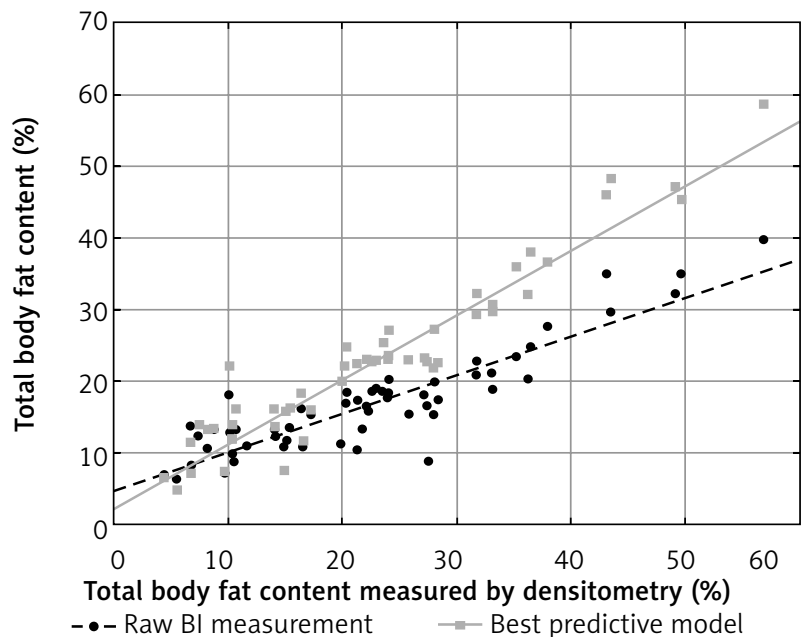

Figure 4. Correlation of raw and model-adjusted bioimpedance values with densitometry results for fatty tissue content within the trunk

compared with densitometry $[6,17,18]$. Studies conducted by Sluytera, Wan, Seo, and ourselves indicate that the rate of underestimation of body fatty tissue content, determined using the BIA method, as compared with DXA, increased with the increased fatty tissue contents [18-21]. Such results were observed in both children and adults. When studying adult, obese women, Neovius has shown a significant underestimation of fatty tissue contents, as determined using the BIA method compared with DXA [22]. Considering the difference between measurements performed using BIA and DXA, a mathematical analysis has been performed as a part of this study to design a model that would reduce the differences between measurements of total fatty tissue contents, obtained using the BIA method, as compared with DXA, with consideration of factors that impact the body's fatty tissue contents.

The fatty tissue distribution in the body depends on ethnic origin, sex, age, and pubertal phase, and its evaluation is considered a risk factor for obesity and its consequences [11-15]. In studies performed by Henche, the trunk fatty tissue mass depended on age and sex (starting from the age of 10, girls have a smaller muscular mass and a greater fatty tissue mass than boys), while fatty tissue contents within the trunk may be a risk factor for obesity [23]. There have been racial differences noted in terms of the distribution of subcutaneous fat between White and Black girls as well as within ethnic groups residing in New Zealand [24,25]. Sex and pubertal phase are factors that influence the trunk fatty tissue contents in other groups of children and youths [26,27]. In studies conducted by Sluyter, it has been noted that there is a correlation between the body fatty tissue contents percentage difference in DXA, BIA measurements, and BMI [20]. In a former study by the same authors, a correlation 
has been shown between the body fatty tissue contents percentage difference, as measured using DXA and BIA, and age, standardized BMI, and standardized waist circumference [18]. These parameters have been taken into account in the multi-factorial regression analysis. However, following statistical analysis, it has been shown that results best correlated with DXA were obtained using a single-factorial model.

A substantially greater difference has been noted when comparing the trunk fatty tissue contents values, as measured using densitometry and bioimpedance. This may be related to different mathematical models defining various parts of the body in DXA and BIA examinations. It has been noted that BIA measurements underestimate the fatty tissue contents in the lower parts of the body and overestimate its contents in the upper part of the body. Therefore, the multi-factorial regression analysis of trunk fatty tissue takes into account not only individual patient features but also the fatty tissue contents in various parts of the body.

As a result, statistical models reducing the bioimpedance measurement error have been presented, as compared with the densitometric result in terms of total and segmental fatty tissue mass contents in children. In our studies, the use of a regression model reduces the differences between results obtained using densitometry and those achieved with bioelectric impedance, with regard to fatty tissue contents evaluation in children, thus improving the measurement accuracy.

There are no previous studies that would allow fatty tissue contents reference values to be determined for BIA. However, American researchers have determined reference values for DXA, including both total and segmental fatty tissue contents $[28,29]$. The use of mathematical models reducing the BIA measurement error, as compared with DXA, will enable results of these measurements to be referenced against DXA reference values.

The evaluation of fatty tissue contents within the trunk using BIA and DXA is increasingly used in the developmental age. Each of these methods has its advantages and disadvantages. Lately, BIA is increasingly used for the measurement of total and segmental fatty tissue mass for the evaluation of excessive weight and obesity therapy results. The use of the proposed statistical model may increase the accuracy of the measurement result using bioelectric impedance, thus reducing the measurement error, as compared with the result obtained with densitometry.

\section{Conclusions}

Bioimpedance underestimates the total fatty tissue contents and fatty tissue contents within the trunk. Use of a multi-factorial regression model reduces the differences between results obtained using densitometry and those achieved with bioelectric impedance, in terms of segmental fatty tissue content evaluation in children, improving the measurement accuracy.

\section{Disclosure}

The authors report no conflict of interest.

\section{References}

1. Ng M, Fleming T, Robinson M, et al. Global, regional and national prevalence of overweight and obesity in children and adults 1980-2013: A systematic analysis. Lancet 2014; 384: 766-781.

2. Xu S, Xue Y. Pediatric obesity: Causes, symptoms, prevention and treatment (review). Exp Ther Med 2016; 11: 15-20.

3. Alves Junior CA, Mocellin MC, Gonçalves ECA, et al. Anthropometric indicators as body fat discriminators in children and adolescents: A systematic review and meta-analysis. Adv Nutr An Int Rev J 2017; 8: 718-727.

4. Siegel MJ, Hildebolt CF, Bae KT, et al. Total and intraabdominal fat distribution in preadolescents and adolescents: Measurement with MR imaging. Radiology 2007; 242: 846-856.

5. Baum T, Cordes C, Dieckmeyer M, et al. MR-based assessment of body fat distribution and characteristics. Eur J Radiol 2016; 85: 1512-1518.

6. Wohlfahrt-Veje C, Tinggaard J, Winther K, et al. Body fat throughout childhood in 2647 healthy Danish children: Agreement of $\mathrm{BMI}$, waist circumference, skinfolds with dual X-ray absorptiometry. Eur J Clin Nutr 2014; 68: 664-670.

7. Chiplonkar S, Kajale N, Ekbote V, et al. Validation of bioelectric impedance analysis against dual-energy. Indian Pediatr 2017; 54: 919-925.

8. Bazzocchi A, Ponti F, Albisinni U, et al. DXA: Technical aspects and application. Eur J Radiol 2016; 85: 1481-1492.

9. Mulasi U, Kuchnia AJ, Cole AJ, Earthman CP. Bioimpedance at the bedside: Current applications, limitations, and opportunities. Nutr Clin Pract 2015; 30: 180-193.

10. Kyle UG, Earthman CP, Pichard C, Coss-Bu JA. Body composition during growth in children: Limitations and perspectives of bioelectrical impedance analysis. Eur J Clin Nutr 2015; 69: 1298-1305.

11. Aucouturier J, Meyer M, Thivel D, et al. Effect of android to gynoid fat ratio on insulin resistance in obese youth. Interact between Dis Concepts Organ Heal Care 2009; 5: 134-137.

12. Lätt E, Mäestu J, Jürimäe J. Longitudinal associations of android and gynoid fat mass on cardiovascular disease risk factors in normal weight and overweight boys during puberty. Am J Hum Biol 2018: e23171.

13. Stults-Kolehmainen MA, Stanforth PR, Bartholomew JB. Fat in android, trunk, and peripheral regions varies by ethnicity and race in college aged women. Obesity 2012; 20: 660-665.

14. Aucouturier J, Meyer M, Thivel D, et al. Effect of android to gynoid fat ratio on insulin resistance in obese youth. Arch Pediatr Adolesc Med 2009; 163: 826-831.

15. Sergi G, De Rui M, Stubbs B, et al. Measurement of lean body mass using bioelectrical impedance analysis: a consideration of the pros and cons. Aging Clin Exp Res 2017; 29: 591-597.

16. Brantlov S, Jødal L, Lange A, et al. Standardisation of bioelectrical impedance analysis for the estimation of body composition in healthy paediatric populations: a systematic review. J Med Eng Technol 2017; 41: 460-479.

17. Thivel D, Verney J, Miguet M, et al. The accuracy of bioelectrical impedance to track body composition changes depends on the 
degree of obesity in adolescents with obesity. Nutr Res 2018; 54: 60-68.

18. Jakubowska-Pietkiewicz E, Prochowska A, Fendler W, Szadkowska A. Porównanie metod pomiaru odsetka tkanki tłuszczowej u dzieci. Pediartic Endocrinol Diabetes Metab 2009: 248-252.

19. Seo Y-G, Kim JH, Kim Y, et al. Validation of body composition using bioelectrical impedance analysis in children according to the degree of obesity. Scand J Med Sci Sports 2018; 28: 2207-2215.

20. Sluyter JD, Schaaf D, Scragg RKR, Plank LD. Prediction of fatness by standing 8-electrode bioimpedance: A multiethnic adolescent population. Obesity 2010; 18: 183-189.

21. Wan CS, Ward LC, Halim J, et al. Bioelectrical impedance analysis to estimate body composition, and change in adiposity, in overweight and obese adolescents: Comparison with dual-energy x-ray absorptiometry. BMC Pediatr 2014; 14: 249.

22. Neovius M, Hemmingsson E, Freyschuss B, Uddén J. Bioelectrical impedance underestimates total and truncal fatness in abdominally obese women. Obesity 2006; 14: 1731-1738.

23. Henche SA, Torres RR, Pellico LG. An evaluation of patterns of change in total and regional body fat mass in healthy Spanish subjects using dual-energy X-ray absorptiometry (DXA). Eur J Clin Nutr 2008; 62: 1440-1448.

24. Rush EC, Scragg R, Schaaf D, et al. Indices of fatness and relationships with age, ethnicity and lipids in New Zealand European, Māori and Pacific children. Eur J Clin Nutr 2009; 63: 627-633.

25. Wagner DR, Heyward VH. Measures of body composition in blacks and whites: a comparative review. Am J Clin Nutr 2000; 71: 1392-1402.

26. He Q, Horlick M, Thornton J, et al. Sex-specific fat distribution is not linear across pubertal groups in a multiethnic study. Obes Res 2004; 12: 725-733.

27. Lim JS, Hwang JS, Cheon GJ, et al. Gender differences in total and regional body composition changes as measured by dual-energy X-Ray absorptiometry in Korean children and adolescents. J Clin Densitom 2009; 12: 229-237.

28. Kelly TL, Wilson KE, Heymsfield SB. Dual energy X-ray absorptiometry body composition reference values from NHANES. PLoS One 2009; 4: e7038.

29. Fan B, Shepherd JA, Levine MA, et al. National Health and Nutrition Examination Survey Whole-Body Dual-Energy X-Ray Absorptiometry Reference Data for GE Lunar Systems. J Clin Densitom 2014; 17: 344-377. 